\title{
Hematomas en el lago escleral: complicación tras esclerectomía profunda no perforante
}

\section{Hematoma in the scleral lake: complication of non-penetrating deep sclerectomy}

\author{
Carmen Navarro-Perea*, Jose L. Torres-Peña y Esperanza Gutierrez-Diaz \\ Servicio de Oftalmología, Hospital Universitario 12 de Octubre, Universidad Complutense de Madrid, Madrid, España
}

\begin{abstract}
Resumen
Método: Presentamos 4 pacientes con glaucoma de ángulo abierto no controlado y cataratas, y un paciente con glaucoma uveítico secundario a heterocromía de Fuchs. Todos ellos fueron intervenidos mediante facoemulsificación y esclerectomía profunda no perforante con 5-fluoracilo. Dos de los pacientes presentaron hematoma en el lago escleral a las $24 \mathrm{~h}$ tras la cirugía, otros 2 a la semana y uno de ellos a las 2 semanas de la intervención. El hematoma se diagnosticó mediante gonioscopia en todos los casos. Además todos presentaron la presión intraocular elevada. Resultado: El hematoma del lago escleral suele aparecer en las 2 primeras semanas tras la cirugía. A todos los pacientes se les realizó goniopuntura en los primeros 5 meses de la complicación y uno de ellos requirió una segunda goniopuntura 3 meses después. A uno de los pacientes se le realizó una cistitomía. Tres de los pacientes requirieron tratamiento con betabloqueantes tópicos para controlar las cifras de presión intraocular. Conclusión: Los hematomas en el lago escleral son una complicación que puede aparecer en las primeras 2 semanas tras una esclerectomía profunda no perforante, pudiendo producir un mal funcionamiento de la cirugía por lo que es necesario la introducción de antihipertensivos oculares para el control de la presión intraocular. La goniopuntura podría ser usada para resolver esta complicación.
\end{abstract}

Palabras clave: Glaucoma. Hematoma en el lago escleral. Esclerectomía profunda no perforante. Goniopuntura. Cistitomía.

\begin{abstract}
Methods: We describe 4 patients suffering from open-angle glaucoma and cataract, and one patient with secondary glaucoma after Fuchs' heterochromic uveitis. All of them underwent phacoemulsification and non-penetrating deep sclerectomy with 5-fluoracil. Two patients presented hematoma in scleral lake $24 \mathrm{~h}$ after surgery, other 2 of them presented it one week after the intervention, and one of them presented it 2 weeks after the surgery. The hematoma was found by gonioscopy in all cases. Moreover, all patients had elevated intraocular pressure. Result: Hematoma usually appears within 2 weeks after surgery. Goniopunctures were performed in 5 patients within 5 months of the complication. A second goniopuncture was needed for one of them. Bleb needling was made in one of them. Three of the patients required treatment with beta-blocker eye drops since intraocular pressure exceeded pre-set values. Conclusion: Hematomas in scleral lake are a rare complication that may appear within 2 weeks after non-penetrating deep sclerectomy. They may cause surgery malfunction and antihypertensive eye drops might be required for tensional control. Goniopuncture could be used for solving the complication.
\end{abstract}

Key words: Glaucoma. Hematoma in the scleral lake. Non-penetrating Deep Sclerectomy. Goniopuncture. Bleb needling.

\footnotetext{
Correspondencia:

*Carmen Navarro-Perea

Fecha de recepción: 01-01-2017

Fecha de aceptación: 12-04-2017

E-mail: carmennavarroperea@gmail.com

DOI: 10.1016/j.mexoft.2017.04.001

Disponible en internet: 01-05-2019

Rev Mex Oftalmol. 2019;93(3):149-152 www.rmo.com.mx

0187-4519/C 2017 Sociedad Mexicana de Oftalmología. Publicado por Permanyer México SA de CV. Este es un artículo Open Access bajo la licencia CC BY-NC-ND (http://creativecommons.org/licenses/by-nc-nd/4.0/).
} 


\section{Introducción}

La esclerectomía profunda no perforante (EPNP) es una técnica de cirugía filtrante antiglaucomatosa que ha demostrado un correcto control de presión intraocular (PIO), con menos complicaciones postoperatorias que la trabeculectomía. La presencia de la membrana trabeculodescemética proporciona un filtrado más controlado, disminuyendo así todas las complicaciones potenciales relacionadas con la apertura de la cámara anterior (hipema, hipotonías, desprendimientos coroideos, inflamación intraocular, endoftalmitis, entre otras) ${ }^{1}$. Dentro de las complicaciones de la EPNP está descrita la presencia de sangre en el lago escleral, que puede comprometer el éxito del procedimiento, sin embargo existe muy poca literatura al respecto.

Presentamos un estudio retrospectivo, evaluado y aceptado por el Comité de Investigación. Se trata de 5 casos con esta complicación, en los que solo en 2 se consiguieron PIO postoperatorias satisfactorias sin tratamiento hipotensor.

\section{Casos clínicos}

\section{Caso clínico 1}

Varón de 48 años sin tratamiento antiagregante ni anticoagulante, con glaucoma uveítico secundario a heretocromía de Fuchs en ojo derecho, con mal control tensional a pesar de tratamiento tópico máximo, por lo que se indicó EPNP con 5-fluoracilo y facoemulsificación, que se realizó sin incidencias. La PIO 24 h tras la cirugía fue de $13 \mathrm{mmHg}$, pero a la semana de la intervención presentó PIO de $36 \mathrm{mmHg}$ con ampolla de filtración aplanada y coloración roja oscura en la zona temporal del colgajo, por lo que se sospechó un hematoma en lago escleral, que se confirmó mediante gonioscopia. Se inició tratamiento tópico con timolol + brimonidina y dexametasona. En las siguientes revisiones la PIO descendió a $10 \mathrm{mmHg}$ y el hematoma desapareció parcialmente. Al mes se retiró el tratamiento hipotensor y la PIO volvió a aumentar $(22 \mathrm{mmHg})$, realizándose goniopuntura, pero sin suficiente descenso de la PIO, ya que se mantuvo en $21 \mathrm{mmHg}$, por lo que fue necesaria la reintroducción de tratamiento tópico hipotensor.

\section{Caso clínico 2}

Varón de 78 años en tratamiento con clopidogrel y diagnosticado de glaucoma primario de ángulo abierto (GPAA), con PIO elevada a pesar de tratamiento médico máximo y atrofia óptica en ojo derecho, en el que se indicó EPNP con 5-fluoracilo y facoemulsificación. La PIO $24 \mathrm{~h}$ tras la cirugía fue de $22 \mathrm{mmHg}$, por lo que se pautó timolol colirio. Dos semanas más tarde, mediante gonioscopia se observó la ocupación del lago escleral por sangre, presentando una PIO de $17 \mathrm{mmHg}$ con tratamiento. La actitud inicial fue expectante y se retiró el tratamiento hipontensor. A los 5 meses, volvió a presentar PIO de $22 \mathrm{mmHg}$ por lo que se realizó goniopuntura, y posteriormente cistitomía, sin suficiente reducción de PIO, siendo necesario la reintroducción del tratamiento tópico hipotensor para mantener las cifras tensionales controladas.

\section{Caso clínico 3}

Varón de 70 años en tratamiento con ácido acetilsalicílico a dosis antiagregante y en seguimiento por GPAA, con cifras tensionales elevadas $(32 \mathrm{mmHg})$ a pesar de tratamiento tópico hipotensor y con excavación papilar del $80 \%$. Se decidió realizar EPNP con 5-fluoracilo. La PIO $24 \mathrm{~h}$ tras la cirugía fue de $6 \mathrm{mmHg}$. Pero a los 15 días el paciente presentó PIO de $37 \mathrm{mmHg}$, con marcada hiperemia conjuntival y sangre en el lago escleral visible mediante gonioscopia, por lo que se inició tratamiento tópico con timolol y se mantuvo corticoterapia tópica. Al mes de la cirugía se realizó revisión de la ampolla con inyección de mitomicina- $C$ subconjuntival $(0.002 \mathrm{mg} / \mathrm{ml})$, sin reducción de la PIO (30 mmHg), por lo que a los 2 meses se hizo una goniopuntura, con buena respuesta tensional (PIO de $14 \mathrm{mmHg}$ ).

\section{Caso clínico 4}

Mujer de 71 años, no antiagregada ni anticoagulada, con GPAA con daño glaucomatoso moderado pero con progresión del mismo y PIO límite a pesar de tratamiento médico $(26 \mathrm{mmHg})$, por lo que se indicó EPNP con 5-fluoracilo y facoemulsificación en ojo derecho. La PIO 24 h tras la cirugía fue de 26 mmHg, observándose sangre en lago escleral. La actitud inicial fue expectante, para favorecer la filtración de humor acuso y así ayudar a la desaparición del hematoma. En las revisiones posteriores se observó disminución de la sangre en el lago escleral (Fig. 1) pero la PIO se mantuvo elevada $(24 \mathrm{mmHg}$ ) por lo que se reintrodujo tratamiento tópico hipotensor y se realizó goniopuntura 2 meses después de la cirugía. Tras la misma la PIO se mantuvo en $22 \mathrm{mmHg}$ por lo que se tuvo que reiniciar tratamiento hipotensor para control de PIO $(15 \mathrm{mmHg})$. 


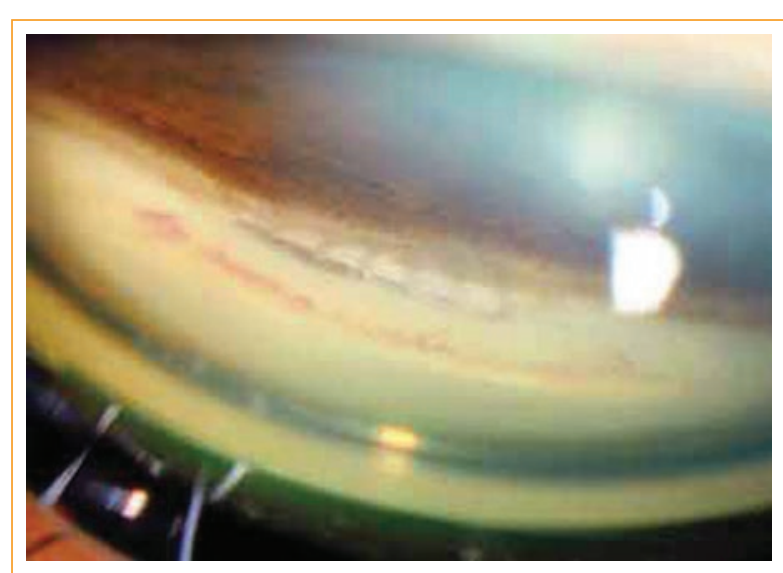

Figura 1. Hematoma en lago escleral en resolución. Imagen de gonioscopia en la que se observa ventana trabeculodescemética libre, con pequeños restos hemáticos en la zona anterior; en la zona posterior puede visualizarse a través de la membrana el extremo del implante esnoper acanalado.

\section{Caso clínico 5}

Mujer de 73 años en tratamiento con ácido acetilsalicílico a dosis antiagregante y en seguimiento por GPAA, con PIO de $26 \mathrm{mmHg}$ y progresión a pesar de tratamiento hipotensor, en la que se realizó EPNP con 5-fluoracilo y facoemulsificación en ojo derecho. La PIO $24 \mathrm{~h}$ tras la cirugía fue de $23 \mathrm{mmHg}$ observándose sangre en lago escleral, por lo que se inició tratamiento tópico con timolol + brimonidina. Se realizó goniopuntura al mes de la cirugía, presentando PIO posteriores controladas $(15 \mathrm{mmHg})$ sin precisar tratamiento tópico hipotensor.

\section{Discusión}

La EPNP asociada a antimetabolitos ha demostrado ser eficaz en la reducción de la $\mathrm{PIO}^{2,3}$ y presenta ventajas sobre la trabeculectomía como son: el mantenimiento de la integridad estructural del ojo, evita una descompresión súbita y permite un retorno más precoz a la actividad física normal ${ }^{4}$. Esto conlleva una reducción de las complicaciones por hiperfiltración, ya que la membrana trabeculodescemética proporciona un sistema de filtración más controlado. Sin embargo, presenta una serie de desventajas potenciales, como es la necesidad de uso de antimetabolitos para mejorar el resultado tensional ${ }^{5}$, además precisa revisiones periódicas, ya que puede aparecer un aumento tardío de la resistencia a la salida del humor acuoso ${ }^{6}$.
Pero la EPNP no está exenta de complicaciones, y entre las principales se encuentran: la rotura de la membrana trabeculodescemética, la hipertensión ocular postoperatoria, la fibrosis de la ampolla, la no identificación del canal de Schlemm y la incarceración de iris $^{7}$. También se pueden producir otras complicaciones comunes con la trabeculectomía, tales como desprendimiento de coroides, hipotonía prolongada, hifema, cámara anterior poco profunda y la prueba de Seidel positiva ${ }^{4}$, pero con una prevalencia más baja.

En nuestra serie de casos presentamos otra complicación, que es la presencia de un hematoma en el lago escleral. Esta complicación ha sido poco descrita en la literatura, y no se ha referido que su presencia influya en el control tensional ${ }^{8}$. En nuestra opinión, la presencia del hematoma podría provocar el mal funcionamiento del procedimiento, ya que puede dificultar un flujo adecuado de humor acuoso a través del lago escleral y la presencia de sangre favorecería la fibrosis precoz del colgajo, con el consecuente aumento de PIO tras la cirugía.

El principal tratamiento empleado en nuestros pacientes ha sido la goniopuntura, con el objetivo de facilitar la evacuación de la sangre hacia la cámara anterior y aumentar la salida de humor acuoso, lo cual ayudaría a la eliminación de los restos de sangre en el lago escleral y la formación de un flujo de salida adecuado. Una vez restablecido el flujo hacia el lago escleral, si la PIO sigue elevada, puede complementarse con una revisión de ampolla para eliminar la fibrosis externa. Tanto la OCT de segmento anterior como la biomicroscopia ultrasónica anterior son valiosas pruebas diagnósticas, siempre que se disponga de ellas, ya que demuestran la existencia o no de ampolla y lago escleral, y si la revisión con aguja puede ser de utilidad o no $0^{9,10}$.

En los 5 pacientes de nuestra serie, el hematoma apareció en los primeros 15 días tras la cirugía, se diagnosticó mediante gonioscopia y en todos los casos provocó un aumento de la PIO. Aunque en las revisiones sucesivas se observó la reabsorción total o parcial del hematoma, la PIO permaneció elevada. En todos ellos se realizó goniopuntura en un intervalo de tiempo variable tras la intervención, y en 2 de ellos también se hizo una revisión con aguja de la ampolla de filtración con mitomicina- $C$ intentado abrir las paredes de la ampolla y levantar el colgajo superficial si era accesible, a pesar de lo cual solo en 2 casos se consiguió la PIO objetivo y en los 3 casos restantes fue necesario reiniciar tratamiento hipotensor. 
Como conclusión, destacamos la posible importancia de los hematomas en el lago escleral como causa de fracaso de la EPNP, por lo que es necesario realizar un diagnóstico y un tratamiento precoz y agresivo para evitar la fibrosis externa. Sin embargo, debido al reducido número de casos, no podemos concluir si la goniopunción y/o cistitomía con inyección de mitomicina-C, son o no eficaces para la resolución de esta complicación.

\section{Responsabilidades éticas}

Protección de personas y animales. Los autores declaran que para esta investigación no se han realizado experimentos en seres humanos ni en animales.

Confidencialidad de los datos. Los autores declaran que han seguido los protocolos de su centro de trabajo sobre la publicación de datos de pacientes.

Derecho a la privacidad y consentimiento informado. Los autores declaran que en este artículo no aparecen datos de pacientes.

\section{Financiamiento}

Los autores no recibieron patrocinio para llevar a cabo este artículo.

\section{Conflicto de intereses}

Los autores declaran no tener ningún conflicto de intereses.

\section{Bibliografía}

1. Ramos López FJ, Francés Muñoz E, López-Sánchez EV, et al. Estimación de la eficacia de la esclerectomía profunda no perforante en el tratamiento quirúrgico del glaucoma al a no de la cirugía. Arch Soc Esp Oftalmol. 2003;78:197-201.

2. Lachkar $Y$, Hamard P. Nonpenetrating filtering surgery. Curr Opin Ophthalmol. 2002;13:110-5.

3. Marek R, Joanna W, Lewczuk K, et al. Efficacy and safety of deep sclerectomy and phacoemulsification and deep sclerectomy in clinical of Military Health Service Institute-yearly observations. Klin Oczna. 2006;108:385-91.

4. Vazi RT, Silvai PC, Colombini GN. Goniosynechiae as a complication of non-penetrating deep sclerectomy by inappropriate use of mydriatic: Case report. Arq Bras Oftalmol. 2008;71:599-600.

5. Shin DH, Iskander NG, Ahee JA, et al. Long-term filtration and visual field outcomes after primary glaucoma triple procedure with and without mitomycin-C. Ophthalmology. 2002;109:1607-11.

6. Muñoz-Negrete FJ, Rebolleda G, Noval S. Facoesclerectomía profunda no perforante. Resultados y complicaciones. Arch Soc Esp Oftalmol. 2003;78:499-506.

7. Roy S, Mermoud A. Complications of deep nonpenetrating sclerectomy. J Fr Ophtalmol. 2006;29:1180-97.

8. Gemma Rebolleda G, Muñoz FJ. Complicaciones de cirugías combinadas: facoemulsificación + trabeculectomía o esclerectomía profunda no perforante. En: Lorente R, Mendicute J, editores. Cirugía del cristalino. Madrid: MAC LINE SL.; 2008. p.1263-73.

9. Bouhéraoua N1, Hamard P, lordanidou V, et al. Assessment of anterior segment anatomy by OCT after non penetrating deep sclerectomy. J Fr Ophtalmol. 2012;35:760-7.

10. Osman EA. The benefit of ultrasound biomicroscopy (UBM) in management of total Descemet's membrane detachment after deep sclerectomy surgery. Int Ophthalmol. 2011;31:345-8. 